\title{
Morfologia de esporos de pteridófitas do Parque Estadual das Fontes do Ipiranga (São Paulo, Brasil) Família: 2-Blechnaceae
}

\author{
Carolina Brandão Coelho ${ }^{1}$ e Luciano Mauricio Esteves ${ }^{1,2}$
}

Recebido: 06.12.2007; aceito: 21.08.2008

ABSTRACT - (Morphology of spores of pteridophytes from the "Parque Estadual das Fontes do Ipiranga" (São Paulo, Brazil), Family: 2 - Blechnaceae). Spores of seven pteridophyte species of the family Blechnaceae from the "Parque Estadual das Fontes do Ipiranga" were studied. The family is represented in the area by two genera: Blechnum and Salpichlaena. Descriptions, illustrations, observations, as well as an identification key are presented.

Key words: Blechnaceae, Blechnum, pteridophyte, Salpichlaena, spore

RESUMO - (Morfologia de esporos de pteridófitas do Parque Estadual das Fontes do Ipiranga (São Paulo, Brasil), Família: 2 - Blechnaceae). Foram estudados os esporos de sete espécies de pteridófitas pertencentes à família Blechnaceae no Parque Estadual das Fontes do Ipiranga (PEFI). A família está representada na área por dois gêneros: Blechnum e Salpichlaena. Para todos os táxons estudados são apresentadas descrições, ilustrações, observações, bem como chave para a identificação das espécies.

Palavras-chave: Blechnaceae, Blechnum, esporo, pteridófitas, Salpichlaena

\section{Introdução}

A família Blechnaceae possui cerca de nove gêneros e 200 espécies no mundo. É uma família que ainda depende de novos estudos para a definição de sua circunscrição, particularmente sobre as relações entre Blechnum s.l. e outros gêneros (Smith et al. 2006). São plantas terrestres, rupícolas, ou às vezes epífitas, hemiepífitas, ou trepadeiras, distribuindo-se, principalmente no hemisfério sul, com uma única espécie (B. spicant (L.) Roth) ocorrendo em regiões temperadas do hemisfério norte (Prado 2004).

É representada na área do Parque Estadual das Fontes do Ipiranga (PEFI) somente por dois gêneros e sete espécies: Blechnum (B. binervatum (Poir.) C.V. Morton \& Lellinger subsp. acutum (Desv.) R.M. Tryon \& Stolze, B. brasiliense Desv., B. imperiale (Fée \& Glaziou) H. Christ, B. occidentale L., B. polypodioides Raddi, B. proliferum Rosenst.) e Salpichlaena (Salpichlaena volubilis (Kaulf.) J. Sm.).

Segundo Tryon \& Lugardon (1990), os esporos são monoletes variando de elipsodais a esferoidais. O perisporo, sob microscopia eletrônica de varredura, apresenta-se com um padrão laminado, ou seja, ocorre uma expansão laminar que acaba formando uma superfície marcante e característica para a família toda, sendo facilmente distinta de outras pteridófitas. O exosporo apresenta-se sempre psilado, sem nenhum tipo de ornamentação.

A análise morfológica dos esporos das espécies de Blechnaceae, presentes no PEFI, complementa os estudos taxonômicos da família e amplia o material de referência para outros estudos palinológicos, tais como os de precipitação esporo-polínica e a palinologia de sedimentos na área.

\section{Material e métodos}

O material utilizado para o estudo da morfologia dos esporos foi obtido de plantas herborizadas depositadas no Herbário Científico do Estado "Maria Eneyda P. Kaulfmann Fidalgo" (SP) (sigla segundo Holmgren et al. 1990). Quando os espécimes apresentavam-se inférteis, ou com quantidade insuficiente de esporos, optou-se por coletas a partir de exsicatas provenientes de outras localidades.

Os esporos foram preparados segundo a técnica de acetólise (Erdtman 1960). As medidas foram feitas em microscopia óptica com esporos acetolisados e fotografados digitalmente utilizando-

1. Instituto de Botânica, Caixa Postal 3005, 01061-970 São Paulo, SP, Brasil

2. Autor para correspondência: luciano.esteves@uol.com.br 
se fotomicroscópio Olympus BX 50 acoplado a uma câmara de vídeo e microcomputador (PC) e o programa Pro-Plus versão 3 para Windows. Foram medidos os diâmetros: equatorial maior (EM) e menor (Em), polar (P) e o comprimento da lesão (L), determinando-se a média, o intervalo de confiança a $95 \%$ e o coeficiente de variabilidade da amostra. As lâminas preparadas encontram-se depositadas na Palinoteca da Seção de Dicotiledôneas, Instituto de Botânica, São Paulo.

A terminologia adotada é a recomendada por Lellinger (2002). O planejamento do estudo da presente flora e os dados referentes ao Parque Estadual das Fontes do Ipiranga (PEFI) encontram-se descritos no trabalho de Melhem et al. $(1981,1984)$. A chave para identificação da família Blechnaceae no PEFI foi publicada em Prado (2004).

\section{Resultados e Discussão}

\section{Blechnum L.}

Esporos monoletes, elipsoidais ou, raramente, esferoidais, com o contorno normalmente formado pelo perisporo. Exosporo de duas camadas, psilado e eventualmente gemado. Perisporo apresenta uma ou duas camadas com superfície folhada ou com dobras proeminentes e esculturadas, ou ainda em cristas.

1. Blechnum binervatum (Poir.) C.V. Morton \& Lellinger ssp. acutum (Desv.) R.M. Tryon \& Stolze

Figuras 1-6

Forma: monolete, elipsoidal, côncavo-convexo.

Lesão: aproximadamente $1 / 2$ do diâmetro equatorial maior

Superfície: Exosporo psilado, com duas camadas de espessura semelhantes. Perisporo com duas camadas, a externa mais compacta, com poucas dobras e levemente granulado.

Medidas $(\mu \mathrm{m}):$ S.A. Nicolau et al. 981: $\mathrm{P}=40,0$ $\pm 0,4 ; \mathrm{EM}=60,4 \pm 0,6 ; \mathrm{Em}=58,4 \pm 0,5 ; \mathrm{L}=31,2$ $\pm 0,4$.

J.R. Pirani et al. 188: $\mathrm{P}=39,7 \pm 0,6 ; \mathrm{EM}=59,1$ $\pm 0,7 ; \mathrm{Em}=59,2 \pm 0.9 ; \mathrm{L}=30,6 \pm 0,6$.

Observações: o material J. Prado \& D.M. Vital 1434, citado por Prado (2004), não foi estudado por apresentar esporos em quantidade insuficiente para a análise.

Material examinado: 21-XI-1982, J.R. Pirani, et al. 188 (SP); 17-XII-1995, S.A. Nicolau et al. 981 (SP).

\section{Blechnum brasiliense Desv.}

Figuras 7-10

Forma: monolete, elipsoidal, plano-convexo.

Lesão: aproximadamente $3 / 4$ do diâmetro equatorial maior

Superfície: Exosporo psilado, formado por duas camadas. Perisporo rugulado-perfurado e facilmente removido pela acetólise.

Medidas $(\mu \mathrm{m}): \mathrm{P}=35,8 \pm 0,4 ; \mathrm{EM}=49,2 \pm 0,5 ;$ $\mathrm{Em}=34,2 \pm 0,5 ; \mathrm{L}=32,2 \pm 0,3$.

Observações: os materiais $O$. Handro s.n. (SP49043), O. Handro 2245 e J. Prado \& D.M. Vital 1423, citados por Prado (2004), não foram estudados por apresentarem esporos em quantidade insuficiente para a análise.

Material examinado: 13-VII-1960, G. Eiten et al. 2083 (SP).

\section{Blechnum imperiale (Fée \& Glaziou) H. Christ}

Figuras 11-15

Forma: monolete, elipsoidal, côncavo-convexo.

Lesão: aproximadamente $3 / 4$ do diâmetro equatorial maior

Superfície: Exosporo psilado, com duas camadas, a mais externa folhada e aproximadamente três vezes mais espessa que a interna. Perisporo laminado, bastante fino e com poucas pregas, aderido ao exosporo, mas eventualmente removido com a acetólise.

Medidas ( $\mu \mathrm{m}): \mathrm{P}=66,8 \pm 1,4 ; \mathrm{EM}=81,8 \pm 1,1 ;$ $\mathrm{Em}=72,8 \pm 1,7 ; \mathrm{L}=53,8 \pm 1,7$.

Material examinado: 18-I-1945, O. Handro s.n. (SP43072).

\section{Blechnum occidentale L.}

Figuras 16-21

Forma: monolete, elipsoidal.

Lesão: aproximadamente $3 / 4$ do diâmetro equatorial maior

Superfície: Exosporo psilado, formado por duas camadas. Perisporo com duas camadas, a interna densa e a externa fina e granulada sem dobras, parcialmente removida com a acetólise.

Medidas ( $\mu \mathrm{m})$ : O. Handro s. $n$. (SP44459): $\mathrm{P}=$ $32,5 \pm 1,2 ; \mathrm{EM}=48,1 \pm 0,9 ; \mathrm{Em}=46,9 \pm 0,6 ; \mathrm{L}=$ $33,5 \pm 0,8$. 

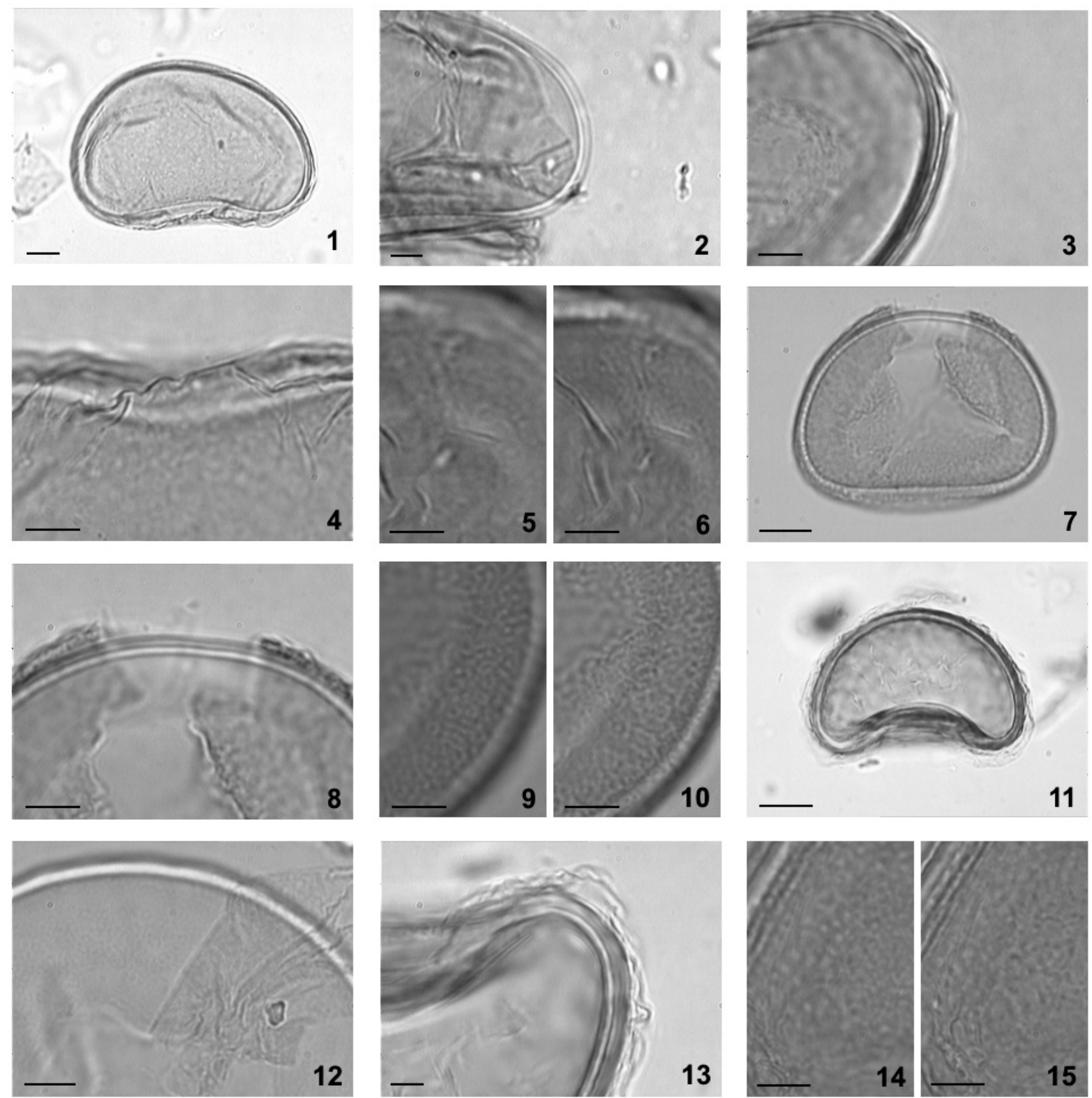

Figuras 1-15. Fotomicrografias de esporos do gênero Blechnum L. (Blechnaceae). 1-6. Blechnum binervatum (Poir.) C.V. Morton \& Lellinger ssp. acutum (Desv.) R.M. Tryon \& Stolze. 1. Vista equatorial. 2. Corte óptico pelo exosporo (seta) e perisporo. 3. Corte óptico da parede, perisporo fraturado (seta). 4. Corte óptico pelo perisporo indicando dobras. 5-6. Análise de L.O., focos alto e baixo, respectivamente. 7-10. Blechnum brasiliense Desv. 7. Vista equatorial, perisporo rugulado-perfurado fraturado, exosporo psilado. 8. Corte óptico pelo exosporo (E) e perisporo (P). 9-10. Análise de L.O., focos alto e baixo, respectivamente. 11-15. Blechnum imperiale (Fée \& Glaziou) H. Christ. 11. Vista equatorial. 12. Corte óptico pelo exosporo (E) e perisporo (P) fraturado. 13. Corte óptico da parede, perisporo laminado (seta). 14-15. Análise de L.O., foco alto e baixo, respectivamente. Barras de escala: 2-6, 8-10, $13=5 \mu \mathrm{m} ; 1,7,12,13-15=10 \mu \mathrm{m} ; 11=20 \mu \mathrm{m}$. 

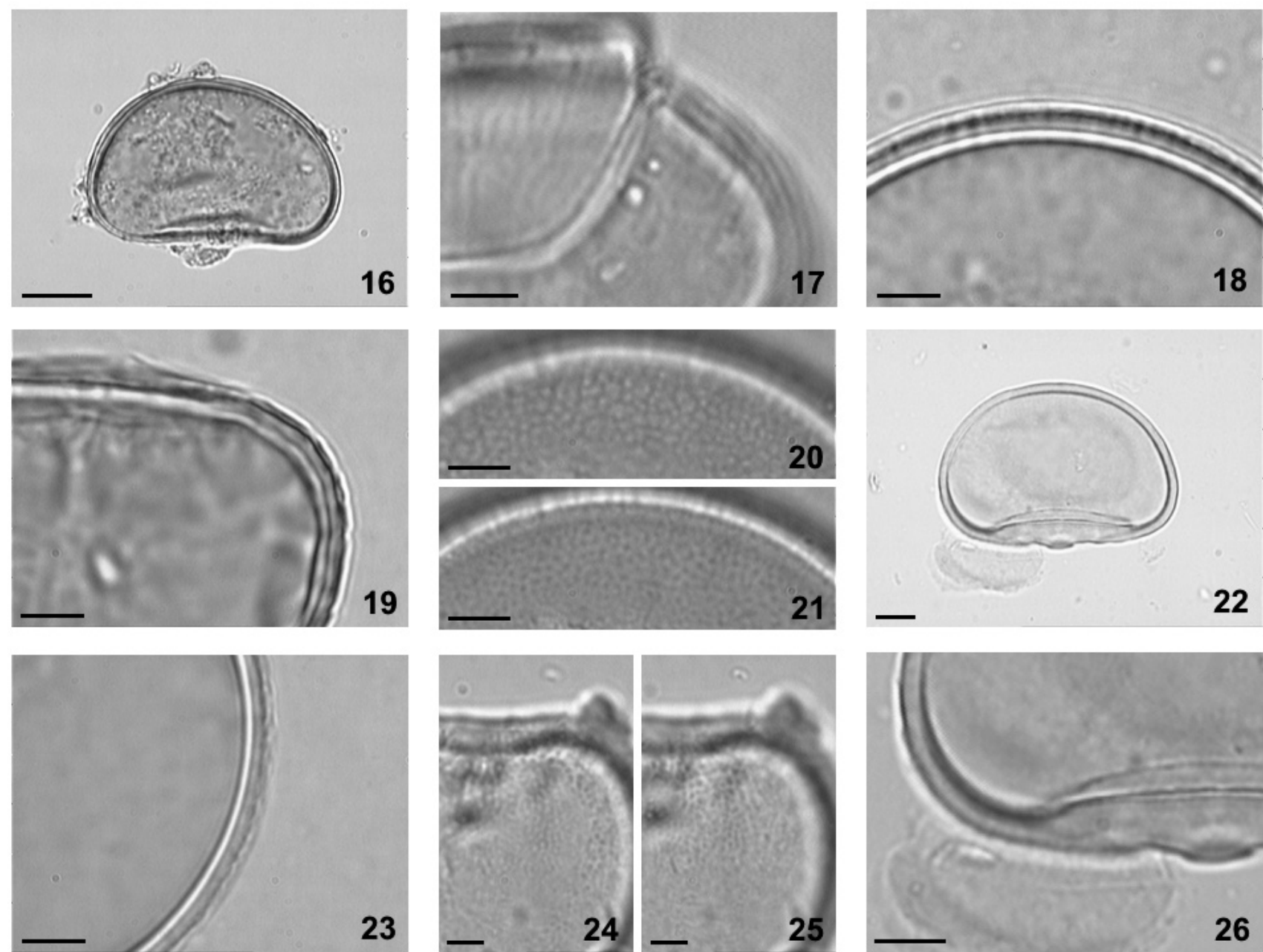

Figuras 16-26. Fotomicrografias de esporos dos gêneros Blechnum L. (Blechnaceae). 16-21. Blechnum occidentale L. 16. Vista equatorial, perisporo fino e granulado (seta). 17. Corte óptico pelo exosporo (E) e perisporo (P) fraturado. 18. Corte óptico da parede. 19. Corte óptico, exosporo com duas camadas. 20-21. Análise de L.O., focos alto e baixo, respectivamente. 22-26. Blechnum polypodioides Raddi. 22. Vista equatorial. 23. Corte óptico, exosporo com duas camadas. 24-25. Análise de L.O., focos alto e baixo, respectivamente. 26. Corte óptico pelo exosporo (E) e perisporo (P) removido. Barras de escala: 18-21, 23-25, 16-17 = 5 $\mu \mathrm{m} ; 22,26=10 \mu \mathrm{m}$.

J.A. Corrêa 54: $\mathrm{P}=31,6 \pm 0,9 ; \mathrm{EM}=46,9 \pm 0,8 ;$ $\mathrm{Em}=44,4 \pm 0,8 ; \mathrm{L}=25,7 \pm 0,5$.

J.A. Corrêa 59: $\mathrm{P}=33,6 \pm 0,5 ; \mathrm{EM}=49,1 \pm 0,5 ;$ $\mathrm{Em}=47,3 \pm 0,4 ; \mathrm{L}=27,0 \pm 0,4$.

J.A. Corrêa 84: $\mathrm{P}=31,8 \pm 0,7 ; \mathrm{EM}=45,6 \pm 0,9 ;$ $\mathrm{Em}=49,4 \pm 0,7 ; \mathrm{L}=26,9 \pm 1,0$.

J.A. Corrêa 125: $\mathrm{P}=32,8 \pm 0,6 ; \mathrm{EM}=44,4 \pm 0,7 ;$ $\mathrm{Em}=47,8 \pm 0,9 ; \mathrm{L}=24,6 \pm 0,4$.

J. Prado \& D.M. Vital 1435: $\mathrm{P}=34,2 \pm 1,0 ; \mathrm{EM}$ $=53,4 \pm 0,8 ; \mathrm{Em}=46,6 \pm 1,1 ; \mathrm{L}=24,0 \pm 0,5$.

Observações: o material W. Hoehne 2439, citado por Prado (2004), não foi estudado por apresentar esporos em quantidade insuficiente para a análise.

Material examinado: 4-VII-1939, O. Handro s.n. (SP44459); 9-IV-1974, J.A. Corrêa 59 (SP); 14-V-
1974, J.A. Corrêa 54 (SP); 18-II-1975, J.A. Corrêa 84 (SP); 9-IV-1976, J.A. Corrêa 125 (SP); 6-X-2003, J. Prado \& D.M. Vital 1435 (SP).

\section{Blechnum polypodioides Raddi}

Figuras 22-26

Forma: monolete, elipsoidal, plano-convexo

Lesão: aproximadamente $1 / 2$ do diâmetro equatorial maior

Superfície: Exosporo psilado formado por duas camadas de difícil visualização sob microscopia óptica. Perisporo fino e fortemente aderido ao exosporo, microrugulado com granulações esparsas. 
Medidas ( $\mu \mathrm{m})$ : O. Handro s.n. (SP44460): $\mathrm{P}=$ $36,6 \pm 1,3 ; \mathrm{EM}=49,7 \pm 1,1 ; \mathrm{Em}=48,2 \pm 1,2 ; \mathrm{L}=$ $25,8 \pm 1,2$.

G. Eiten \& L.T. Eiten 2101: $\mathrm{P}=36,5 \pm 1,1 ; \mathrm{EM}=$ $53,5 \pm 1,0 ; \mathrm{Em}=51,5 \pm 1,4 ; \mathrm{L}=27,5 \pm 0,8$.

G. Eiten \& L.T. Eiten $2108 B: \mathrm{P}=38,7 \pm 0,9 ; \mathrm{EM}$ $=58,6 \pm 1,0 ; \mathrm{Em}=51,5 \pm 0,8 ; \mathrm{L}=27,3 \pm 0,7$.

S.L. Jung et al. $201: \mathrm{P}=38,6 \pm 1,0 ; \mathrm{EM}=55,9 \pm$ 0,$8 ; \mathrm{Em}=51,7 \pm 0,8 ; \mathrm{L}=27,1 \pm 1,1$.

N.A.Rosa \& J.M.Pires 3787: $\mathrm{P}=39,4 \pm 0,6 ; \mathrm{EM}$ $=57,5 \pm 0,8 ; \mathrm{Em}=52,7 \pm 0,6 ; \mathrm{L}=31,0 \pm 0,5$.

J. Prado \& D.M. Vital 1424: $\mathrm{P}=35,1 \pm 0,4 ; \mathrm{EM}$ $=51,9 \pm 0,5 ; \mathrm{Em}=50,6 \pm 0,5 ; \mathrm{L}=29,1 \pm 0,5$.

J. Prado \& G.B. Silva 1414: $\mathrm{P}=32,5 \pm 0,4 ; \mathrm{EM}$ $=47,4 \pm 0,5 ; \mathrm{Em}=47,4 \pm 0,7 ; \mathrm{L}=24,1 \pm 1,1$.

Observações: os materiais G. Eiten \& L.T. Eiten 2065, G. Eiten \& L.T. Eiten 2106, W. Hoehne 2609, J.A. Corrêa 136, J. Prado \& D.M. Vital 1436 citados por Prado (2004), não foram estudados por apresentarem esporos em quantidade insuficiente para a análise.

Material examinado: 4-VII-1939, O. Handro s.n. (SP44460); 14-VII-1960, G. Eiten \& L.T. Eiten 2101 (SP); 14-VII-1960, G. Eiten \& L.T.Eiten 2108B (SP); 14-XI-1977, S.L. Jung et al. 201 (SP); 17-IX-1980, N.A.Rosa \& J.M.Pires 3787 (SP); 6-X-2003, J. Prado \& D.M. Vital 1424 (SP); 10-VI-2003, J. Prado \& G.B. Silva 1414 (SP).

\section{Blechnum proliferum Rosenst.}

Figuras 27-32

Forma: monolete, elipsoidal.
Lesão: aproximadamente $3 / 4$ do diâmetro equatorial maior

Superfície: exosporo psilado, com duas camadas. Perisporo microrugulado com projeções ao redor do contorno do esporo.

Medidas $(\mu \mathrm{m}): \mathrm{P}=58,1 \pm 1,0 ; \mathrm{EM}=77,0 \pm 0,9 ;$ $\mathrm{Em}=68,4 \pm 1,2 ; \mathrm{L}=57,8 \pm 1,0$.

Observações: o material $O$. Handro 2151 , citado por Prado (2004), não foi estudado por apresentar esporos em quantidade insuficiente para a análise.

Material examinado: 8-X-1945, W. Hoehne 1882 (SP).

\section{Salpichlaena Hook.}

\section{Salpichlaena volubilis (Kaulf.) J. Sm.}

Figuras 33-37

Forma: monolete, elipsoidal.

Lesão: aproximadamente $4 / 5$ do diâmetro equatorial maior

Superfície: exosporo psilado com duas camadas. Perisporo rugulado com grânulos e esférulas dispersas irregularmente na superfície.

Medidas $(\mu \mathrm{m}): \mathrm{P}=97,2 \pm 2,3 ; \mathrm{EM}=123,7 \pm 2,2 ;$ $\mathrm{Em}=127,0 \pm 2,5 ; \mathrm{L}=111,1 \pm 3,1$.

Observações: o material W. Hoehne 2645, citado por Prado (2004), não foi estudado por apresentar esporos em quantidade insuficiente para a análise.

Material examinado: 16-X-2003, J. Prado \& D.M. Vital 1441 (SP).

\section{Chave para as espécies de Blechnaceae}

1. Perisporo granulado

2. Presença de esférulas na superfície do perisporo

Salpichlaena volubilis

2. Ausência de esférulas na superfície do perisporo

3. Perisporo micro-rugulado com granulações esparsas

Blechnum polypodioides

3. Perisporo sem micro-rúgulas

4. Perisporo com dobras Blechnum binervatum

4. Perisporo fino e granulado, sem dobras Blechnum occidentale

1. Perisporo sem granulações

5. Perisporo laminado Blechnum imperiale

5. Perisporo não laminado

6. Perisporo micro-rugulado com projeções ao redor do contorno do esporo .... Blechnum proliferum

6. Perisporo rugulado-perfurado, facilmente removido pela acetólise Blechnum brasiliense 

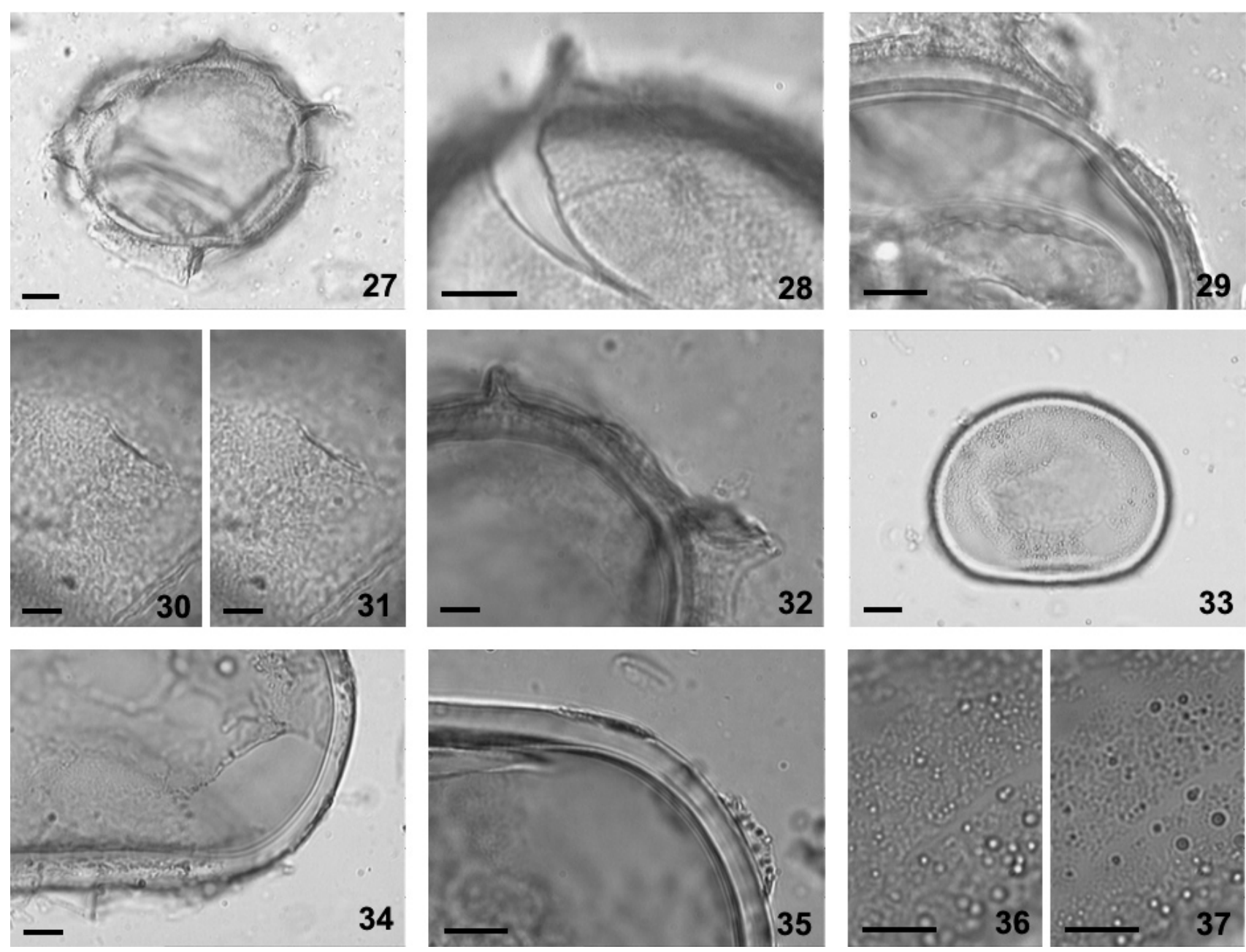

Figuras 27-37. Fotomicrografias de esporos dos gêneros Blechnum L. e Salpichlaena Hook. 27-32. Blechnum proliferum Rosenst. 27. Vista equatorial. 28. Corte óptico pelo exosporo (E) e perisporo (P) fraturado. 29. Corte óptico da parede, exosporo (E) com duas camadas e perisporo (P) fraturado. 30-31. Análise de L.O., focos alto e baixo, respectivamente. 32. Corte óptico da parede, projeções ao redor do contorno (seta). 33-37. Salpichlaena volubilis (Kaulf.) J.Sm. 33. Vista equatorial. 34. Corte óptico da parede, perisporo fraturado (P) e exosporo psilado (E). 35. Corte óptico pelo exosporo e perisporo. 36-37. Análise de L.O., focos alto e baixo, respectivamente; setas indicam as esférulas. Barras de escala: 30-32 $=5 \mu \mathrm{m} ; 27-29,34-37=10 \mu \mathrm{m} ; 33=20 \mu \mathrm{m}$.

\section{Agradecimentos}

Os autores agradecem ao Dr. Jefferson Prado pela identificação do material e ao CNPq pela bolsa PIBIC concedida à primeira autora.

\section{Literatura citada}

Erdtman, G. 1960. The acetolysis method. A revised description. Svensk Botanisk Tidskrift 39: 561-564.

Holmgren, P., Holmgren, N.H. \& Barnett, L.C. 1990. Index herbariorum. 8 ed. New York Botanical Garden, New York.
Lellinger, D.B. 2002. A modern multilingual glossary for Taxonomic Pteridology. Pteridologia n. 3. American Fern Society, Washington.

Melhem, T.S., Giulietti, M., Forero, E., Barroso, G.M., Silvestre, M.S.F., Jung, S.L., Makino, H., Fiuza-deMelo, M.M.R., Chiea, S.C., Wanderley, M.G.L., Kirizawa, M. \& Muniz, C. 1981. Planejamento para a elaboração da "Flora Fanerogâmica da Reserva do Parque Estadual das Fontes do Ipiranga (São Paulo, Brasil)". Hoehnea 9: 63-74.

Melhem, T.S., Makino, H., Silvestre, M.S.F., Cruz, M.A.V. \& Jung-Mendaçolli, S. 1984. Planejamento para a elaboração da "Flora Polínica da Reserva do Parque Estadual das Fontes do Ipiranga (São Paulo, Brasil)". Hoehnea 11: 1-7. 
Prado,J.2004. Criptógamos do Parque Estadual das Fontes do Ipiranga, São Paulo, SP. Pteridophyta: chave para as famílias: 2. Blechnaceae. Hoehnea 31: 1-10.

Smith, A.R., Pryer, K.M., Schuettpelz, E., Korall, P., Schneider, H., Wolf, P.G. 2006. A classification for extant ferns. Taxon 55: 705-731.

Tryon, A.F. \& Lugardon, B. 1990. Spores of the Pteridophyta. Surface, wall structure, and diversity based on electron microscope studies. Springer-Verlag, New York. 